
Not for reproduction, distribution or commercial use.

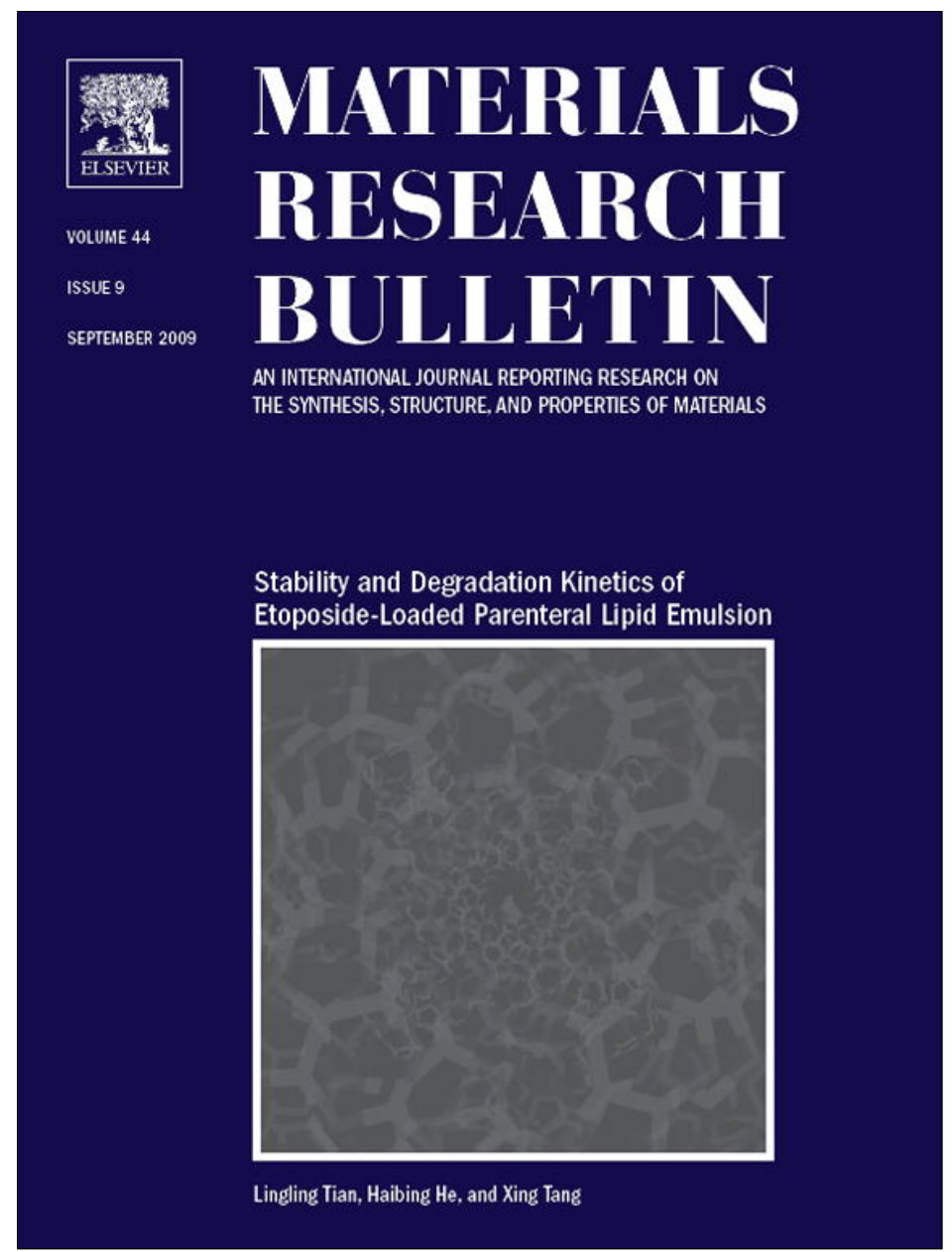

This article appeared in a journal published by Elsevier. The attached copy is furnished to the author for internal non-commercial research and education use, including for instruction at the authors institution and sharing with colleagues.

Other uses, including reproduction and distribution, or selling or licensing copies, or posting to personal, institutional or third party websites are prohibited.

In most cases authors are permitted to post their version of the article (e.g. in Word or Tex form) to their personal website or institutional repository. Authors requiring further information regarding Elsevier's archiving and manuscript policies are encouraged to visit:

http://www.elsevier.com/copyright 


\title{
Effect of activation/purification of multiwalled carbon nanotubes (MWCNTs) on the activity of non-platinum based hypo-hyper d-electrocatalysts for hydrogen evolution
}

\author{
Perica Paunovića,* ${ }^{a}$, Aleksandar T. Dimitrov ${ }^{a}$, Orce Popovski ${ }^{a}$, Evelina Slavcheva ${ }^{\mathrm{b}}$, \\ Anita Grozdanov ${ }^{a}$, Elefteria Lefterova ${ }^{b}$, Gjorgji Petruševski ${ }^{c}$, Svetomir Hadži Jordanov ${ }^{a}$ \\ ${ }^{a}$ Faculty of Technology and Metallurgy, University "Sts. Cyril and Methodius", Skopje, Republic of Macedonia \\ ${ }^{\mathrm{b}}$ Institute of Electrochemistry and Energy Systems, Bulgarian Academy of Sciences, Sofia, Bulgaria \\ ${ }^{\mathrm{c}}$ Research E Development, ALKALOID AD, Aleksandar Makedonski 12, Skopje, Republic of Macedonia
}

A R T I C L E I N F O

\section{Article history:}

Received 3 November 2008

Received in revised form 21 April 2009

Accepted 29 May 2009

Available online 6 June 2009

\section{Keywords:}

MWCNTs

Activation/purification

Catalyst support

Hydrogen evolution

\begin{abstract}
A B S T R A C T
Commercial multiwalled carbon nanotubes (MWCNTs) were used as a catalyst support for non-platinum hypo-hyper d-electrocatalysts. In order to improve the performance of these catalysts, activation/ purification of MWCNTs in acid medium $\left(\mathrm{HNO}_{3}\right)$ was carried out. The physical and surface changes of MWCNTs were investigated by DTA/TGA analysis, Raman spectroscopy, and cyclic voltammetry. Structural changes of the electrocatalysts were observed by infrared spectroscopy and SEM. Their use as a support for electrocatalysts for hydrogen evolution was demonstrated, and shown to be more suitable compared to the traditional carbon support material - carbon black (Vulcan XC-72). The electrocatalysts consisted of $10 \% \mathrm{Co}+18 \% \mathrm{TiO}_{2}+$ MWCNTs. Activation/purification removes the amorphous carbon phase in the MWCNTs. As a result of both shortening and opening of carbon nanotubes, better dispersion of metallic particles (the active catalytic centers) was achieved. Thus, trans-particle and inter-particle porosity of the electrocatalytic material was improved, implying increase of catalytic activity for hydrogen evolution.
\end{abstract}

(c) 2009 Elsevier Ltd. All rights reserved.

\section{Introduction}

One of the most significant segments of the "hydrogen economy" is the choice of an electrode material that satisfies some very strict criteria, such as catalytic activity for hydrogen evolution/oxidation, chemical and mechanical stability, and low price. The most widely used electrode material for hydrogen electrolysers/fuel cells is nano-scaled platinum deposited on carbon black (Vulcan XC-72). However, the commercial use of this material is limited due to its low abundance and high cost. The present Pt resources are estimated to cover only $\sim 20 \%$ of the automotive industry needs [1]. Hence, it is imperatives to reduce or even replace platinum from hydrogen electrolysers/fuel cells. There are two basic approaches to achieve this goal: (i) increase the real surface area of the electrocatalysts by lowering the particle size of both the catalytic and the support phase and (ii) prepare non-platinum multicomponent electrocatalysts with catalytic activity comparable to that of Pt.

\footnotetext{
* Corresponding author. Tel.: +389 75303 579; fax: +389 23064392

E-mail address: pericap@tmf.ukim.edu.mk (P. Paunović).
}

The first (physical) approach introduces nano-scaled catalytic and support components into the modern electrocatalysts. Nanotechnologies and nanomaterials are exceptional tools for modern electrocatalysis. Carbon nanotubes (CNTs), with their unique and superior physical, mechanical and electric properties [2-4], have been used as support materials for electrocatalysts designed for hydrogen electrolysers/fuel cells. Their extraordinary conductivity, mechanical stability and inner geometry makes them also suitable for catalyst support, replacing traditional carbon blacks. In comparison with Vulcan XC-72, which has an electronic conductivity of $4 \mathrm{~S} \mathrm{~cm}^{-1}$, the corresponding value for CNTs is significantly higher at $10^{4} \mathrm{~S} \mathrm{~cm}^{-1}$ [1]. Also, the surface characteristics of CNTs are more appropriate. CNTs possess high specific surface area of 200-900 $\mathrm{m}^{2} \mathrm{~g}^{-1}$ [1]. Due to their geometry (empty cylinders), CNTs have high inter- and trans-particle porosity that provides better dispersion of active catalytic phases over the catalyst's surface.

The second (chemical) approach involves mixing of nonplatinum metals or their compounds in order to achieve activity of the catalysts similar to that of Pt. The so-called "hypo-hyper d-concept" is very important for the development of multicomponent electrocatalysts. According to Jakšić's interpretation [5-7] of Brewer's valence-bond theory [8], a mixture of a metal 
from the right side of the transition series (hyper d-) with a metal (or its compound) from the left side of transition series (hypo d-) does show significant synergetic effect for the electrocatalytic activity.

In our previous works [9-11], we investigated nano-scale hypohyper d-electrocatalysts containing $\mathrm{Ni}$, Co or NiCo as hyper dphase and anatase titania as hypo d-phase, both deposited on carbon substrate. These multiwalled carbon nanotubes (MWCNTs) were compared to traditional carbon black (Vulcan XC-72) as carbon substrates. A considerable increase of catalytic activity for hydrogen evolution was observed as a consequence of the better surface characteristics of the MWCNTs. A Co-TiO 2 -MWCNTs was shown to be the best catalytic system.

However, the prepared MWCNTs always contained other carbonaceous particles such as amorphous carbon, fullerenes, nanoparticles [12-14]. Hence, further treatment of the carbon nanotubes is needed, to separate and remove these particles from the MWCNTs. One of the most used purification/activation methods is treatment by nitric acid [15-17]. During this purification process, shortening and opening of the MWCNTs occurs, creating more active surface. The aim of this work was to improve the electrocatalytic activity of the Co-based hypo-hyper d-electrocatalyst through the activation/purification of manufactured MWCNTs used as catalyst support. The activation/purification was performed into nitric acid and was assisted by mixing of the solution and further filtration.

\section{Experimental}

The manufactured MWCNTs (Guangzhou Yorkpoint Energy Company, China) were activated with $28 \%$ (mass.) $\mathrm{HNO}_{3}$. The solution was prepared with ultrapurified and deionized water. MWCNTs were dispersed into the solution and stirred by magnetic stirrer $(900 \mathrm{rpm})$ for $4 \mathrm{~h}$. Suspended MWCNTs were filtered by vacuum through a micro-filter and further dried at $95^{\circ} \mathrm{C}$ for $12 \mathrm{~h}$. Both activated and manufactured (non-activated) MWCNTs were further investigated by TGA/DTA analysis and Raman spectroscopy in order to identify structural changes caused by the activation/ purification.

The thermal stability of the carbon nanotubes samples was measured using a Perkin Elmer PYRIS Diamond Thermogravimetric/Differential Thermal Analyzer. Ten milligrams of carbon nanotubes were heated in the temperature interval of $25-1000{ }^{\circ} \mathrm{C}$ with a heating rate of $20^{\circ} \mathrm{C} \mathrm{min}^{-1}$, in nitrogen atmosphere.

Raman spectra were obtained using powder samples on a Micro-Raman multichannel spectrometer-Horiba JobinYvon LabRam Infinity $(f \times 10)$ using $532 \mathrm{~nm}$ YAG laser.

Surface changes were observed by change of the ratio of real versus geometric surface area $S_{\mathrm{R}} / S_{\mathrm{G}}$, i.e. roughness of the electrodes prepared by the manufactured and activated MWCNTs. $S_{\mathrm{R}} / S_{\mathrm{G}}$ was determined as a quotient of the catalyst's double layer capacity $C_{\mathrm{dl}}$ versus the double layer capacity $C_{\mathrm{dlo}}$ of a pure oxide surface. The double layer capacity was measured by cyclic voltammetry $[18,19]$. The measurements were performed in alkaline solution in the region of potentials where only charging of the double layer occurs, with a scanning rate of $1-10 \mathrm{mV} \mathrm{s}^{-1}$. Electrochemical AMEL equipment (Function Generator AMEL 568, Potentiostate/Galvanostate 2053 and software package SOFTASSIST 2.0) was used. Gasdiffusion electrodes were prepared out of catalyst's powder by hot pressing at $300^{\circ} \mathrm{C}$ with the back layer consisting of carbon black acetylene and PTFE, and the front layer made out the catalyst and PTFE [20]. The counter electrode was a platinum wire, the reference one- $\mathrm{Hg} / \mathrm{HgO}$, while the electrolyte was a solution of 3.5 M KOH (p.a., Merck) in deionizated ultrapurified water. During the electrochemical testing, the solution in the cathodic area was purged with hydrogen.
Electrocatalysts were prepared by sol-gel procedure described elsewhere [21]. They consist of $10 \%$ (mass.) hyper d-metallic phase (Co), and $18 \%$ hypo d-oxide phase $\mathrm{TiO}_{2}$ deposited on carbon substrate-manufactured and activated MWCNTs. Organometallics were used as precursors of catalyst's components (Me 2,4pentanedionat, $\mathrm{Me}\left(\mathrm{CH}_{3} \mathrm{COCHCOCH}_{3}\right)_{2}$, Alfa Aesar, Johnson Matthey, $\mathrm{GmbH}$ and Ti-isopropoxide, $\mathrm{Ti}\left[\mathrm{OCH}\left(\mathrm{CH}_{3}\right)_{2}\right]_{4}$, Aldrich, 97\%). For a referent electrocatalyst, traditional Pt deposited on Vulcan XC-72 was prepared by the same procedure.

The structure of hypo-hyper d-electrocatalysts was observed by Scanning Electron Microscope (SEM), JEOL, model JEM CX. To determine the level of hypo-hyper d-interaction, infrared spectroscopy was used. The FTIR spectra were recorded using $\mathrm{KBr}$ pellets at room temperature with a Perkin-Elmer System 2000 interferometer. The electrocatalytic activity was measured as described above. Polarization curves were obtained by the steady-state galvanostatic method.

\section{Results and discussion}

\subsection{Raman spectroscopy}

During the purification of MWCNTs by nitric acid functional carboxylic groups are produced $[17,22,23$ ] which enable solubilization of the carbon nanotubes, or more precisely, formation of the well dispersed colloidal suspension [23]. The oxidation rate of the MWCNTs is slower than that of amorphous carbon and other carbonaceous particles [24]. The carboxylic groups surround both the outer and inner walls of the MWCNTs within the suspension, so removal of some metal impurities is possible [17]. These phenomena occurring during the purification/activation of MWCNTs can be successfully detected by means of Raman spectroscopy and TGA/DTA analysis.

Shown in Fig. 1 are Raman spectra of the manufactured and activated MWCNTs. In order to obtain the precise positions (frequencies of occurrence) and maximum intensities of the characteristic Raman bands in the spectra of the investigated samples, a curve fitting procedure was employed (GRAMS32 for Microsoft Windows, Vers. 4.10, Galactic Industries Corporation, 1991-1996). All bands were fitted using a mixed Gaussian-Lorentz function, and a solution converged to minimum after a maximum of 50 iterations. Linear function was used for baseline corrections. The fitted values are shown in Table 1. Both spectra (Fig. 1) are composed of two characteristic lines for nanotubes: $\mathrm{D}$ and $\mathrm{G}$. D-line with a maximum of near $1350 \mathrm{~cm}^{-1}$ indicates disordered carbon atoms, defects such as pentagons and heptagons in graphite, edges of the graphite crystal, and amorphous carbon [25,26]. G-line with maximum at position of $1585 \mathrm{~cm}^{-1}$ is related to the highly oriented graphite [27]. In both cases, a shoulder appears to the right side of the $\mathrm{G}$-line near $1620 \mathrm{~cm}^{-1}$ and denotes a $\mathrm{D}^{\prime}$-peak. The appearance of the $\mathrm{D}^{\prime}$-peak indicates that these carbon nanotubes are multiwalled [12,28]. So, the ratio of intensities of the characteristic peaks $\left(I_{\mathrm{D}} / I_{\mathrm{G}}\right)$ indicates the extent of defects and impurities in the nanotubes.

After activation/purification of the MWCNTs the value of the $I_{\mathrm{D}} / I_{\mathrm{G}}$ ratio decreases from 1384 to 1283 (see Table 1). This means that the amount of defects and other carbonaceous phases are reduced. Relatively low decrease of $I_{\mathrm{D}} / I_{\mathrm{G}}$ ratio suggests that the relative amount of amorphous carbon remains unchanged, i.e. the removed amorphous carbon is compensated by its continuous generation [23]. The treatment with nitric acid, while producing functional carboxylic groups, generates amorphous carbon by shortening and thinning of the MWCNTs, causing increase in both the defect sites and real surface area. This is suitable for increase of MWCNTs reactivity and catalytic activity. After certain time the rate of amorphous carbon generation is higher than its removal, and 


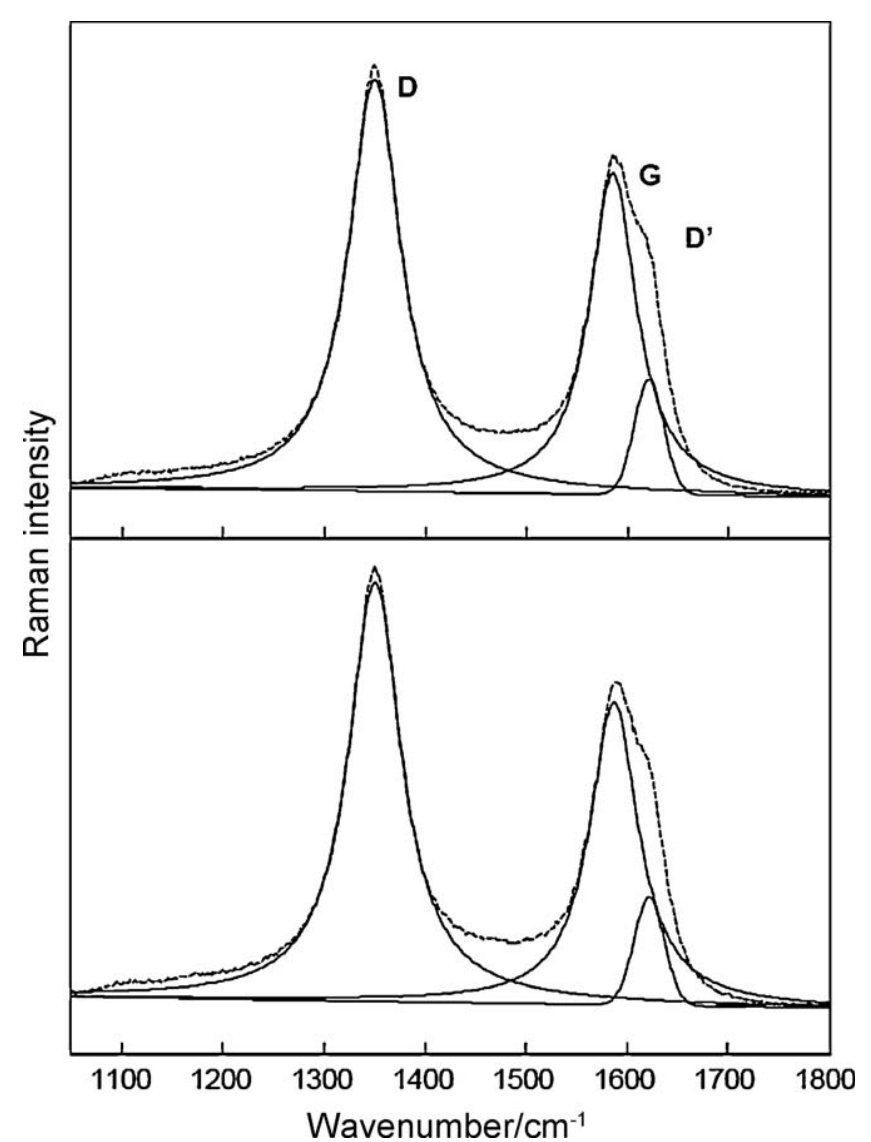

Fig. 1. Raman spectra of the (a) activated and (b) manufactured MWCNTs. The dashed curve presents the real Raman spectra, while the full curves present the outcome of the curve fitting procedure.

consequently the $I_{\mathrm{D}} / I_{\mathrm{G}}$ increases. According to the results of Rosca et al. [23] $I_{\mathrm{D}} / I_{\mathrm{G}}$ starts to increase after $12 \mathrm{~h}$ of oxidation in nitric acid. In this case amorphous carbon generation is slower than its removal.

\subsection{TGA/DTA analysis}

Considering the fact that it is very difficult to separate all the effects and influences during the activation/purification process, the thermal stability of the carbon nanotubes is a good measure for determination of their overall quality. TGA oxidation temperatures $T_{0}$ are basic indicators of the thermal stability of the nanotubes, which depends on several parameters. Namely, nanotubes of smaller diameter oxidize at lower temperature range (lower $T_{0}$ ). Also, defects and derivatization motion into nanotubes, as well as presence of amorphous-like material in both sides of their walls, can cause lower thermal stability. The active metal particles present in the nanotubes are able to catalyze the carbon oxidation, so the amount of metal impurity in the sample can have a considerable influence on the thermal stability of the MWCNTs. Higher $T_{0}$ is always related to purer and less-defective samples. Also, derivative curve temperature $-\mathrm{d} m / \mathrm{d} T_{\max }$ shows well defined DTG peaks related to the carbon combustion rate.
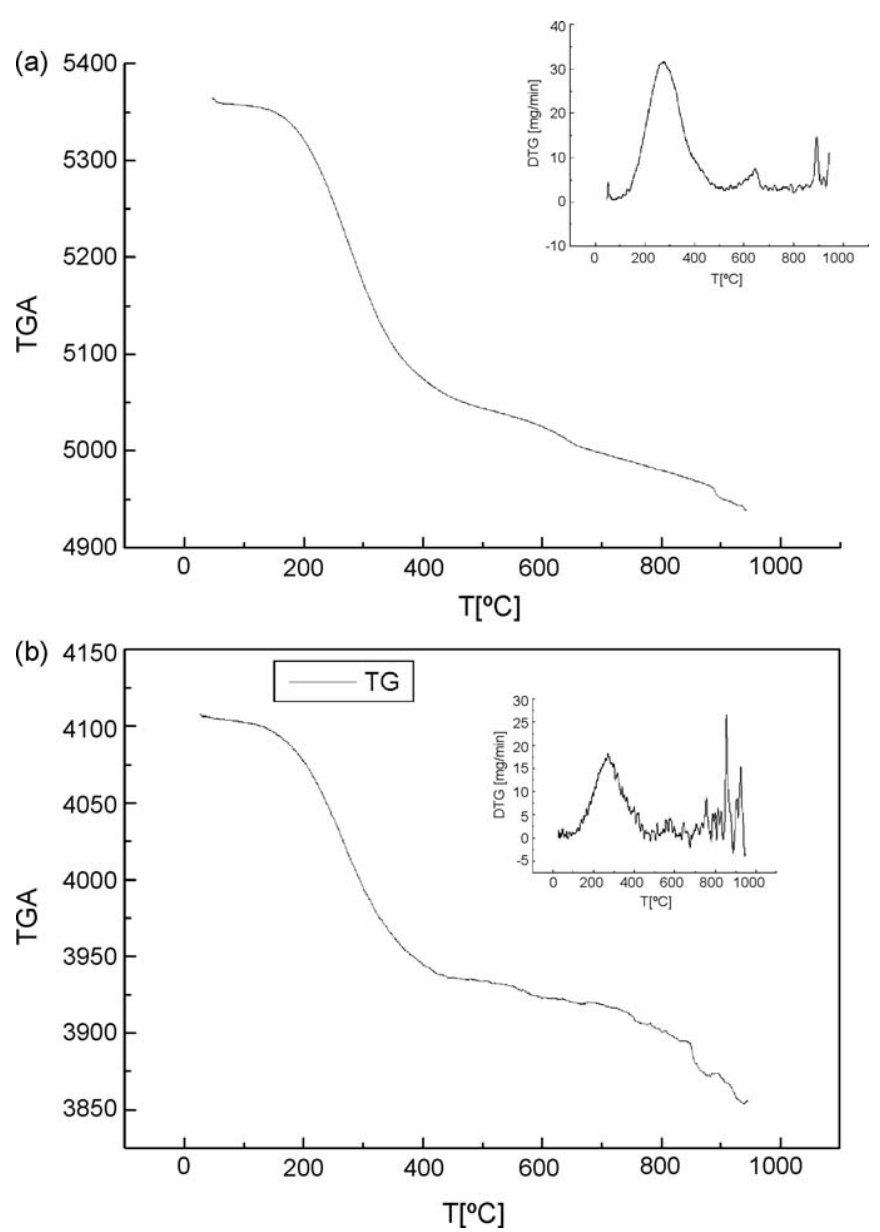

Fig. 2. TGA diagrams of (a) activated and (b) manufactured MWCNTs.

The TGA graphs of the prepared and purified MWCNTs samples are shown in Fig. 2. The characteristic oxidation temperatures and the maxima of the differential thermogravimetric curves (DTG peaks) for the MWCNTs samples are presented in Tables 2 and 3.

According to the values of $T_{0}$ in Table 2 , reduction of weight of the manufactured MWCNTs begins at $523^{\circ} \mathrm{C}$, with complete evaporation at $617^{\circ} \mathrm{C}$. The corresponding temperatures of purified MWCNTs are higher $-585^{\circ} \mathrm{C}$ and $847^{\circ} \mathrm{C}$ respectively. The DTG peak at $575{ }^{\circ} \mathrm{C}$ for the manufactured MWCNTs is assumed to be the optimal combustion temperature of amorphous carbon, while the maximum of the DTG peak at $757^{\circ} \mathrm{C}$ is denoted as combustion temperature of the carbon nanotubes. The corresponding combustion temperatures of amorphous carbon and carbon nanotubes for purified MWCNTs are $645^{\circ} \mathrm{C}$ and $893^{\circ} \mathrm{C}$. The purified MWCNTs show higher values for all characteristic temperatures during their combustion in air, indicating that purified MWCNTs are more stable toward thermal oxidative destruction. The manufactured MWCNTs have smaller diameter and higher presence of defects in the structure. Also, the gradual onset indicates that manufactured MWCNTs contain higher amount of amorphous carbon and for this sample, $T_{0}$, describes

Table 1

Fitted position and maximum intensities of the characteristic peaks in the Raman spectra of activated and as-prepared MWCNTs.

\begin{tabular}{|c|c|c|c|c|c|c|c|}
\hline \multirow[t]{2}{*}{ Sample } & \multicolumn{3}{|c|}{$\begin{array}{l}\text { Fitted position of the characteristic Raman bands } \\
\left(\mathrm{cm}^{-1}\right)\end{array}$} & \multicolumn{4}{|c|}{ Fitted maximum intensities of the characteristic Raman bands } \\
\hline & $\mathrm{D}$ & G & $\mathrm{D}^{\prime}$ & $I_{\mathrm{D}}$ & $I_{\mathrm{G}}$ & $I_{\mathrm{D}}$ & Intensity ratio, $I_{\mathrm{D}} / I_{\mathrm{G}}$ \\
\hline Activated MWCNTs & 1350.173 & 1585.767 & 1621.392 & 1630.868 & 1270.744 & 455.603 & 1.283 \\
\hline Manufactured MWCNTs & 1350.316 & 1586.613 & 1621.347 & 1374.781 & 993.556 & 353.221 & 1.384 \\
\hline
\end{tabular}


Table 2

Characteristic TGA oxidation temperatures of the studied carbon nanotubes samples.

\begin{tabular}{llll}
\hline Sample & $T_{\mathrm{o} 1}\left({ }^{\circ} \mathrm{C}\right)$ & $T_{\mathrm{o} 2}\left({ }^{\circ} \mathrm{C}\right)$ & $T_{\mathrm{o} 3}\left({ }^{\circ} \mathrm{C}\right)$ \\
\hline Activated MWCNTs & 180 & 585 & 847 \\
Manufactured MWCNTs & 173 & 523 & 617
\end{tabular}

Table 3

Characteristic DTG maxima of the studied carbon nanotubes.

\begin{tabular}{llll}
\hline Sample & $T_{\mathrm{o} 1}\left({ }^{\circ} \mathrm{C}\right)$ & $T_{\mathrm{o} 2}\left({ }^{\circ} \mathrm{C}\right)$ & $T_{\mathrm{o} 3}\left({ }^{\circ} \mathrm{C}\right)$ \\
\hline Activated MWCNTs & 278 & 645 & 893 \\
Manufactured MWCNTs & 272 & 575 & 757 \\
\hline
\end{tabular}

the properties of the impurities rather than those of the carbon nanotubes.

\subsection{Real versus geometric surface area}

To assign the surface roughness (the ratio of real versus geometrical surface area, $\left.S_{\mathrm{R}} / S_{\mathrm{G}}\right)$, the double layer capacity $\left(C_{\mathrm{dl}}\right)$ of the catalysts was determined by cyclic voltammetry. The measurements were performed in the region of potentials where only charging of double layer occurs with scanning rate from 1 to $10 \mathrm{mV} \mathrm{s}^{-1}$. The procedure for determination of $C_{\mathrm{dl}}$ for carbon blacks and carbon nanotubes, as well as electrocatalysts deposited on these carbon substrates, is presented elsewhere [29]. The values for the double layer capacity for the manufactured and purified MWCNTs are shown in Table 4. For comparison, the value of $C_{\mathrm{dl}}$ for carbon black (Vulcan XC-72) [29] is also shown. Purified carbon nanotubes have higher value for the double layer capacity, i.e. they have more developed surface area. This value increases from $331 \mathrm{mF} \mathrm{cm}^{-2}$ for manufactured MWCNTs to 342 for purified MWCNTs. As already shown above, this is result of opening, shortening, and thinning of the nanotubes by $\mathrm{HNO}_{3}$ treatment. It should be mentioned that according to the $C_{\mathrm{dl}}$ values, MWCNTs show almost two times higher surface area than the traditionally used carbon substrate, Vulcan XC-72.

\subsection{SEM analysis}

Shown in Fig. 3 are SEM images of the electrocatalysts deposited on manufactured (Fig. 3a and b) and activated MWCNTs (Fig. 3c). The detail shown in Fig. 3a is an aggregation of the metallic (Co) and oxide $\left(\mathrm{TiO}_{2}\right)$ phase, while in Fig. 3b a detail of MWCNTs out of catalytic phases is shown. This suggests that there is no so uniform dispersion of the hypo and hyperd-phases over the carbon substrate. The presence of catalytic phases and MWCNTs is clearly visible in Fig. 3c. This is a characteristic detail representative of the entire observed sample, suggesting that in this case there is better dispersion of the catalytic phase over the substrate. Better dispersion enables larger number of active catalytic centers available for the electrocatalytic reaction of hydrogen evolution. Also, by comparing the length of the MWCNTs, it can be seen that activated MWCNTs are shorter than manufactured MWCNTs. This is a result of the treatment into $\mathrm{HNO}_{3}$ and it contributes to more developed and active surface area for the whole electrocatalyst.

Table 4

Double layer capacity, $C_{\mathrm{dl}}$ of the studied MWCNTs samples.

\begin{tabular}{ll}
\hline Sample & $C_{\mathrm{dl}}\left(\mathrm{mF} \mathrm{cm}^{-2}\right)$ \\
\hline Activated MWCNTs & 342 \\
Manufactured MWCNTs & 331 \\
Vulcan XC-72 [29] & 179 \\
\hline
\end{tabular}

\subsection{Infrared spectroscopy}

Considering the previous results, a higher electrocatalytic activity of the catalyst deposited on activated MWCNTs is expected as a result of the higher surface area. However, electrocatalytic activity can also be improved as a result of the increase of both the surface area (size effect) and intrinsic catalytic activity (intrinsic effect). In order to delineate the intrinsic effect, FTIR analysis was performed.

Infrared spectra of both electrocatalysts deposited on manufactured and activated MWCNTs are shown in Fig. 4. The only band of interest is that by $\mathrm{TiO}_{2}$, positioned at $495 \mathrm{~cm}^{-1}[11,30]$. To determine the hypo-hyper d-interaction, the $\mathrm{TiO}_{2}$ band from the electrocatalyst and the band from pure $\mathrm{TiO}_{2}$ obtained in identical conditions as that in electrocatalyst need to be compared. The peaks of the $\mathrm{TiO}_{2}$ bands originated by the electrocatalysts are shifted to higher wave number $\left(965 \mathrm{~cm}^{-1}\right)$ related to the peak of pure $\mathrm{TiO}_{2}$. The higher the shift of the wave number, the shorter the bond between $\mathrm{TiO}_{2}$ and hyper d-metallic phase, i.e. the higher the hypo-hyper d-interaction. This shift is even $470 \mathrm{~cm}^{-1}$ for both systems. This is an evidence for existence of hypo-hyper dinteraction $\left(\mathrm{Co}-\mathrm{TiO}_{2}\right)$ which causes changes in adsorption characteristics of the electrode materials, and synergetic electrocatalytic effect for the hydrogen evolution. But, the same value of the $\mathrm{TiO}_{2}$ maximum shift for both catalysts deposited on manufactured and activated MWCNTs suggests that after activation of the MWCNTs there is no increase of the interaction between the metallic and oxide phases. Thus, the improvement of the electrocatalytic activity of the studied electrocatalyst can be attributed to the size effect caused by activation/purification of MWCNTs in $\mathrm{HNO}_{3}$.

\subsection{Electrocatalytic activity}

Shown in Fig. 5 are the polarization curves of the studied Cobased electrocatalysts in the plot of current density-overpotential $(i-\eta)$. For comparison, the polarization curve for traditional Pt electrocatalyst deposited on Vulcan XC-72 is shown. This electrocatalyst was prepared in identical conditions as the studied Co-based electrocatalysts. Co-based electrocatalyst deposited on activated MWCNTs shows better catalytic characteristics than that deposited on manufactured MWCNTs. At a reference current density of $60 \mathrm{~mA} \mathrm{~cm}^{-1}$, hydrogen evolution occurs at an overpotential of $215 \mathrm{mV}$ on electrocatalyst deposited on activated MWCNTs, while on electrocatalyst deposited on manufactured MWCNTs it occurs at $235 \mathrm{mV}$. The electrocatalytic activity of the catalyst deposited on activated MWCNTs is very close to (or even better) that of traditional Pt catalyst deposited on Vulcan XC-72. At the same reference current density, the overpotential of hydrogen evolution on traditional Pt electrocatalyst is $220 \mathrm{mV}$. It should be mentioned that the non-platinum electrocatalyst-Co- $\mathrm{TiO}_{2}-$ MWCNTs(a) approaches and even exceeds the catalytic activity of the platinum electrocatalyst-Pt/Vulcan XC-72. This considerable increase of the catalytic activity of Co-based catalyst is result of both intrinsic activity and size effect. As result of introducing $\mathrm{TiO}_{2}$ in the catalyst, hypo-hyper d-interaction $\left(\mathrm{Co}-\mathrm{TiO}_{2}\right.$ interaction) arises, and improves adsorption characteristics of the catalyst, and consequently, causes a synergetic rise of the intrinsic electrocatalytic activity (intrinsic effect). On the other side, introducing MWCNTs as a carbon substrate, considerably improves the real surface area (see Table 4) and porosity of the catalyst (size effect).

For illustration, it was shown in our previous work [11] that, in same experimental conditions, hydrogen evolution overpotential measured at $\mathrm{Co}-\mathrm{TiO}_{2} / \mathrm{MWCNTs}(\mathrm{a})$ was $165 \mathrm{mV}$ lower than that of Co/Vulcan XC-72 electrocatalyst (analogue to Pt/Vulcan XC-72) and $160 \mathrm{mV}$ lower than that of Pt/Vulcan XC-72. 
(a)

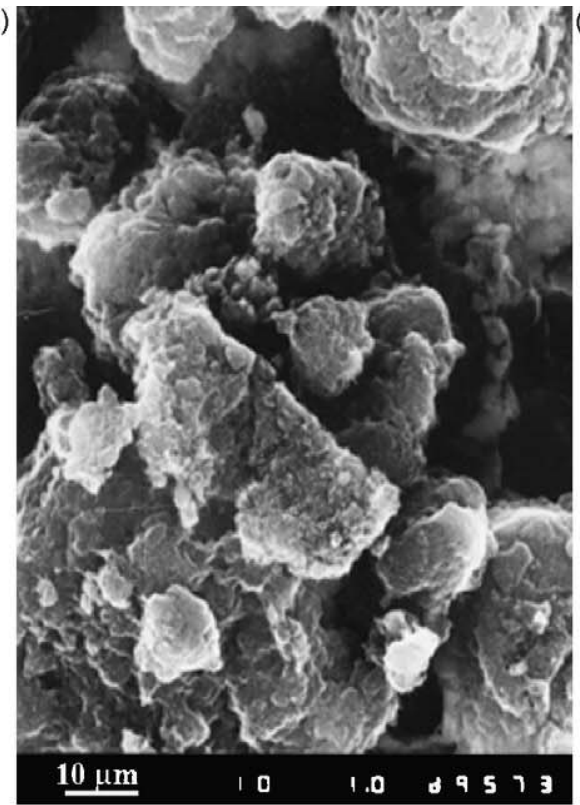

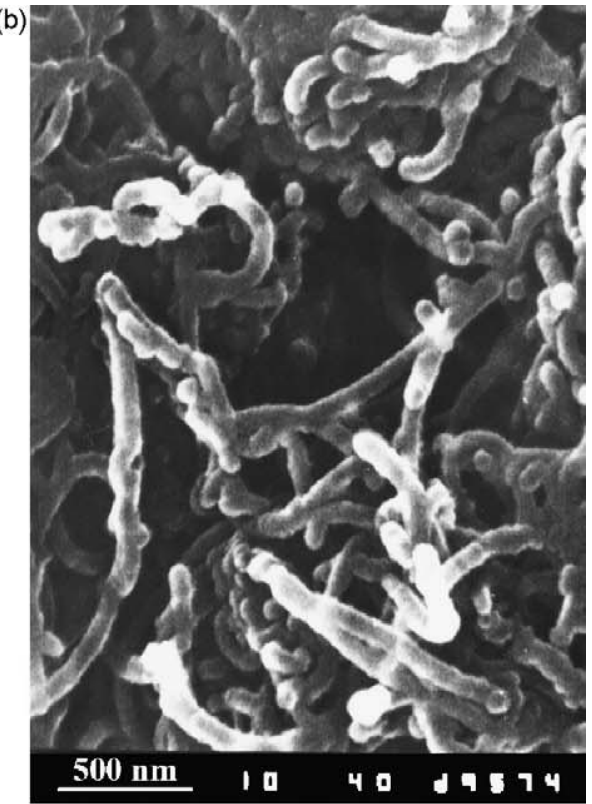

(c)

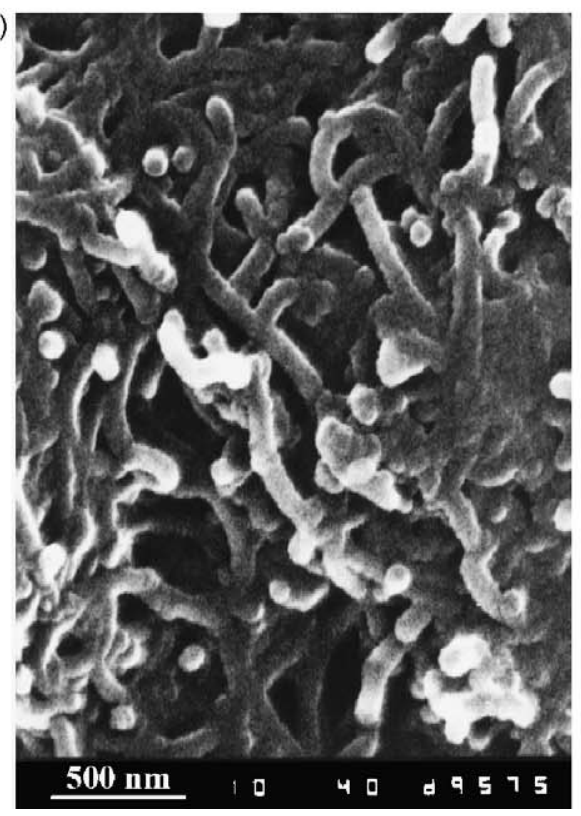

Fig. 3. SEM images of (a and b) prepared and (c) manufactured MWCNTs.

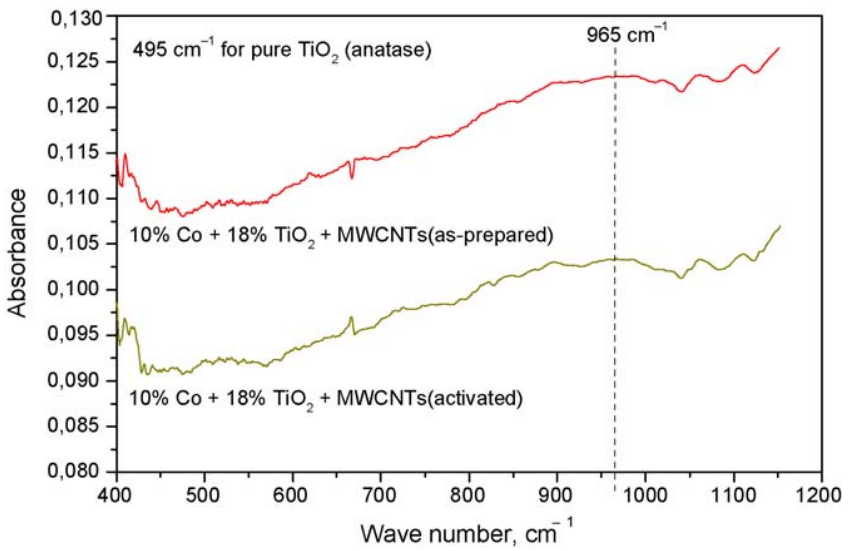

Fig. 4. FTIR spectra of the electrocatalyst with common composition: $10 \% \mathrm{Co}+18 \%$ $\mathrm{TiO}_{2}+$ MWCNTs deposited on manufactured and activated MWCNTs.
The above confirms that remarkable improvement of catalytic activity is achieved by introducing $\mathrm{TiO}_{2}$ and MWCNTs in the Cobased catalyst and by further activation of MWCNTs.

The confirmation of this order of electrocatalytic activities of the studied electrocatalysts are the polarization curves presented in the $\log i-\eta$ plot, shown in Fig. 6. Tafel slopes of the electrocatalysts were determined in the region of $\log i-1$ to 1 . The values determined as indicators for electrocatalytic activity are in agreement with the polarization curves shown in Fig. 5. The Tafel slope for the electrocatalyst deposited on manufactured MWCNTs has a value of $\sim 50 \mathrm{mV}$ decade $^{-1}$ and suggests that this catalyst has lower activity related to the others studied. The Tafel slopes for the electrocatalyst deposited on activated MWCNTs and traditional Pt/Vulcan XC-72 electrocatalyst have very close values $\sim 30 \mathrm{mV}$ decade ${ }^{-1}$.

The improved electrocatalytic activity of the catalyst deposited on activated MWCNTs is a result of the size effect, i.e. more developed real surface area of the catalyst's carbon support after 


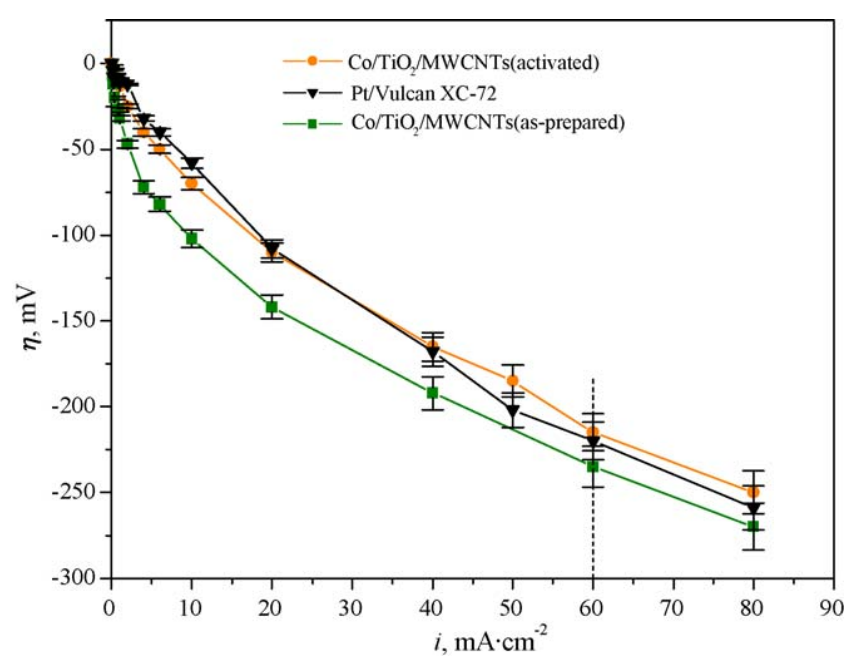

Fig. 5. Polarization curves of the studied electrocatalysts in the plot $i-\eta$.

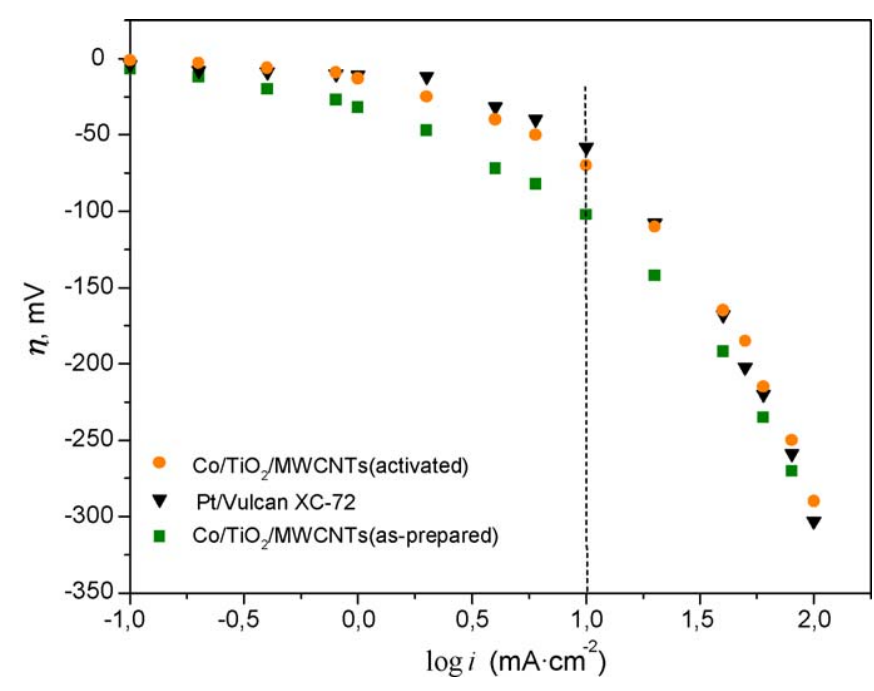

Fig. 6. Polarization curves of the studied electrocatalysts in the plot $\log i-\eta$.

the activation and purification process. As already discussed, besides the amorphous carbon removing, the process results in shortening and opening of the nanotubes and consequently results in an increase of the defective sites. All of these contribute to the increase of the MWCNTs surface area and reactivity that is caused by the higher surface area of the whole Co-based hypo-hyper delectrocatalyst. Increase of the intrinsic catalytic activity was not detected according to the results of infrared spectroscopy.

\section{Conclusion}

The investigations in this study were motivated by the idea to produce non-platinum electrocatalyst $\left(10 \% \mathrm{Co}+18 \% \mathrm{TiO}_{2}+\right.$ MWCNTs) for hydrogen evolution with catalytic activity close to or better than that of Pt. In this stage, activation/purification of the carbon substrate (MWCNTs) was performed in $\mathrm{HNO}_{3}$ in order to promote electrocatalytic activity of the catalyst. According to the achieved result, we can draw the following conclusions:

1. During activation/purification of MWCNTs, two main processes occur: (i) removing of other carbonaceous phases such as amorphous carbon and (ii) shortening, thinning and opening of the MWCNTs, followed by amorphous carbon generation. According to the Raman spectra and TGA/DTA diagrams it can be concluded that the first process is dominant. Activated MWCNTs are purer and more stable. Opening and shortening of the MWCNTs, and the increased number of defective sites as results of the amorphous carbon generation, results in increase of their real surface area and reactivity, characteristics suitable for carbon substrates used for electrocatalysts.

2. Activation/purification of MWCNTs contributes to higher catalytic activity for hydrogen evolution of the Co-based hypo-hyper d-electrocatalyst. This activity is very close to that of the traditional Pt electrocatalyst (Pt/Vulcan XC-72). The increase of the electrocatalytic activity is due to the increased real surface area of the carbon substrate (MWCNTs) after the activation process. The intensity of hypo-hyper d-interaction was the same in both electrocatalysts deposited on manufactured and activated MWCNTs, so there is no increase of the intrinsic activity.

\section{Acknowledgements}

This work was supported by and carried out within the Bilateral Project of the Ministry of Education and Science of Macedonia with the Ministry of Science of Bulgaria. Great gratitude to Dobrin Nedelkov, Intrinsic Bioprobes Inc., Tempe, Arizona, for language improvement of the manuscript. In the course of publishing of this paper, Academician E. Budevski passed away, but he will be remembered as a leading scientist and extraordinary person.

\section{References}

[1] K. Lee, J. Zhang, H. Wang, D.P. Wilkinson, J. Appl. Electrochem. 36 (2002) 507.

[2] T.D. Burchell (Ed.), Carbon Materials for Advanced Technologies, Elsevier Science Ltd., 1999.

[3] M. Endo, S. Iijima, M.S. Dresselhaus (Eds.), Carbon Nanotubes, Elsevier Science Limited, 1996.

[4] K. Tanaka, T. Yamabe, K. Fukui (Eds.), The Science and Technology of Carbon Nanotubes, Elsevier Science Ltd., 1999.

[5] M.M. Jakšić, Int. J. Hydr. Energy 12 (1987) 727

[6] M.M. Jakšić, Mater. Chem. Phys. 22 (1989) 1.

[7] M.M. Jakšić, J. New Matter. Electrochem. Syst. 3 (2000) 153.

[8] L. Brewer, in: P. Rudman, J. Stringer, R.I. Haffee (Eds.), Phase Stability in Metals and Alloys, McGraw-Hill, New York, 1967, p. 39.

[9] P. Paunović, O. Popovski, S. Hadži Jordanov, A. Dimitrov, D. Slavkov, J. Serb. Chem. Soc. 71 (2006) 149.

[10] P. Paunović, O. Popovski, A. Dimitrov, D. Slavkov, E. Lefterova, S. Hadži Jordanov, Electrochim. Acta 52 (2006) 1610.

[11] P. Paunović, O. Popovski, A. Dimitrov, D. Slavkov, E. Lefterova, S. Hadži Jordanov, Electrochim. Acta 52 (2007) 4640.

[12] Y.S. Park, Y.C. Choi, K.S. Kim, D.C. Chung, D.J. Bae, K.H. An, S.Ch. Lim, X.Y. Zhu, Y.H. Lee, Carbon 39 (2001) 655.

[13] D.T. Colbert, J. Zhang, S.M. McClure, P. Nikolaev, Z. Chen, J.H. Hafner, D.W. Owens, P.G. Kotula, C.B. Carter, J.H. Weaver, A.G. Rinzler, R.E. Smalley, Science 266 (1994) 1218.

[14] Y. Ando, S. Iijima, Jpn. J. Appl. Phys. 32 (1993) L107.

[15] C.M. Chen, M. Chen, F.C. Leu, S.Y. Hsu, S.C. Wang, S.C. Shi, C.F. Chen, Diamond Relat. Mater. 14 (2005) 798.

[16] A.G. Rinzler, J. Liu, H. Dai, P. Nikolaev, C.B. Huffman, F.J. Rodrigez-Macias, P.J. Boul, A.H. Lu, D. Heymann, D.T. Colbert, R.S. Lee, J.E. Fisher, A.M. Rao, P.C. Eklund, R.E. Smalley, Appl. Phys. A 67 (1998) 29.

[17] P. Serp, M. Corrias, P. Kalck, Appl. Catal. A: Gen. 253 (2003) 337.

[18] B. Marsan, N. Fradette, G. Beaudoin, J. Electrochem. Soc. 139 (1992) 1889.

[19] L.M. Da Silva, L.A. De Faria, J.F.C. Boodts, Electrochim. Acta 47 (2001) 395

[20] BG patent appl. 38581 (1978).

[21] S. Hadži Jordanov, P. Paunović, O. Popovski, A. Dimitrov, D. Slavkov, Bull. Chem. Technol. Macedonia 23 (2004) 101.

[22] A. Kuznetsova, I. Popova, J.T. Yates, M.J. Bronikowski, C.B. Huffman, J. Liu, R.E. Smalley, H.H. Hwu, J.G. Chen, J. Am. Chem. Soc. 123 (2001) 10699.

[23] I.D. Rosca, F. Watari, M. Uo, T. Akasaka, Carbon 43 (2005) 3124.

[24] T.W. Ebbesen, P.M. Ajayan, H. Hiura, K. Tanigaki, Nature 367 (1994) 148.

[25] H. Hiura, T.W. Ebbesen, K. Tanigaki, H. Takahashi, Phys. Chem. Lett. 202 (1993) 509.

[26] W. Li, H. Zhang, C. Wang, L. Xu, K. Zhu, S. Xie, Appl. Phys. Lett. 70 (1997) 2684.

[27] F. Tunistra, J.L. Koenig, J. Chem. Phys. 53 (1970) 1126.

[28] M.A. Pimenta, A. Marucci, S.A. Empedocles, M.G. Bewendi, E.B. Hanlon, A.M. Rao, P.C. Eklund, R.E. Smalley, G. Dresselhaus, M.S. Dresselhaus, Phys. Rev. B 58 (1998) R16016.

[29] P. Paunović, A.T. Dimitrov, O. Popovski, D. Slavkov, S. Hadži Jordanov, Maced. J. Chem. Chem. Eng. 26 (2007) 87.

[30] P. Paunović, Ph.D. Thesis, University “Sts. Cyril and Methodius”, Skopje, 2005, p. 96. 